\title{
Resolution Correction for Surface X-ray Diffraction at High Beam Exit Angles
}

\author{
By C. Schamper, H. L. Meyerheim and W. Moritz \\ Institut für Kristallographie und Mineralogie der Universität München, Theresienstrasse 41, 80333 München, \\ Germany
}

(Received 23 September 1992; accepted 29 April 1993)

\begin{abstract}
Owing to the two-dimensional periodicity of a superstructure on the crystal surface, the intensity in reciprocal space is continuously distributed along rods normal to the sample surface. The analysis of rod scans in surface $\mathrm{X}$-ray diffraction provides information about the structure parameters normal to the sample surface. For high resolution to be achieved, the measurements must extend to momentum transfers $q_{\perp}$ that are as large as possible. At large exit angles, the conventional Lorentz factor must be modified to take account of the finite aperture of the detector and the continuous intensity along the lattice rod. For two types of $Z$-axis diffractometer used in surface X-ray crystallography, an analytical expression for the resolution correction of rod-scan intensity data has been developed. It takes into account an anisotropic detector resolution $T(\Delta \Theta, \Delta \Phi)$, the finite width of the diffracted beam and the primary-beam divergence parallel to the sample surface, $\Delta \tau$. The calculation of the convolution functions is simplified by a projection onto the $q_{\perp}=0$ plane. The effects of different detector settings and the influences of the primary-beam divergence and the sample quality on the measured intensity are demonstrated for several examples.
\end{abstract}

\section{Introduction}

Grazing-incidence X-ray diffraction has become a successful technique of surface atomic-structure analysis. Recently, several complex structures of reconstructed surfaces have been solved using the standard methods of X-ray crystallography, such as the Patterson function, Fourier synthesis and leastsquares refinement (Feidenhans'l, 1989; Robinson, 1991).

Since a superstructure at the crystal surface is nonperiodic normal to the sample surface, the intensity of the superlattice reflections is continuously distributed along $q_{\perp}=l \times c^{*}$, the component of the momentum transfer $\mathbf{q}$ normal to the sample surface. Most of the experiments performed so far have focused on the measurement of in-plane intensities

(C) 1993 International Union of Crystallography

Printed in Great Britain - all rights reserved $\left(q_{\perp} \simeq 0\right)$, only providing information about the projected structure. On the other hand, only the analysis of the intensity distribution along the superlattice rods provides depth-resolved information. For high resolution to be achieved, the measurements must extend to high-momentumtransfer $q_{\perp}$; this condition can be satisfied by using either large exit angles or large incident angles. We refer here to the case of grazing incidence and large exit angles that is often used.

The scattering geometry is shown in Fig. 1 in real and reciprocal space. The sample is illuminated by the incoming X-ray beam, $\mathbf{k}_{i 0}$, at grazing incidence. The incidence angle $\alpha_{i}$ is of the order of magnitude of $\alpha_{c}$, the critical angle of total external reflection. In many cases, $\alpha_{i}$ is chosen to be even smaller, in order to reduce the background.

The integrated intensity $E\left(\mathbf{Q}_{0}\right)$ of the reflection related to the scattering vector $\mathbf{Q}_{0}=\mathbf{k}_{f 0}-\mathbf{k}_{i 0}$ is measured by rotating the sample around the surface normal $\mathbf{n}$, while the detector is kept constant at the position corresponding to the scattered beam, $\mathbf{k}_{f 0}$. However, for the measurement along the specular direction $\left(00 q_{\perp}\right)$, different procedures must be used which are not considered here.

The scattering vector $\mathbf{Q}_{0}$ can be separated into $Q_{0 \perp}$ and $Q_{0 \|}$, the out-of-plane and in-plane components, respectively. It is easy to see that $Q_{0 \perp}=\left|\mathbf{k}_{f 0}\right| \sin \alpha_{f}$, where $\alpha_{f}$ is the beam exit angle.

For the measurement of out-of-plane intensities, several diffractometer setups have been developed. One convenient way to have access to large beam exit angles at a constant grazing-incidence angle is realized in the ' $Z$-axis' machine developed by Brennan \& Eisenberger (1984). In Fig. 2, two realizations of $Z$-axis diffractometers are shown that emphasize the different detector arrangements. In both cases, the detector has two degrees of freedom as compared with one degree of freedom in a four-circle machine.

In the first design (Fig. 2a), the detector can be rotated around the surface normal by the $\Theta$ axis, which coincides with the $y$ axis. Note that this axis is also denoted $2 \Theta$ in the literature, however we chose $\Theta$ in order to have a unique assignment for each type of diffractometer. An exit angle $\Phi_{0}\left(\Phi_{0}=\alpha_{f}\right)$ is

Journal of Applied Crystallography ISSN 0021-8898 $\pi ; 1993$ 
adjusted by rotating the detector around the axis $\Phi$ that lies in the crystal-surface plane and is perpendicular to the scattering vector. This kind of diffractometer (hereinafter called the type- $A$ diffractometer) is used, for example, by the Risø group at the Hamburger Synchrotron Strahlungs Labor (HASYLAB). The angle calculations for this geometry have been given by Bloch (1985).

The second type of diffractometer, which is hereinafter called the type- $B$ diffractometer, has been developed in our group (Kretschmar, Wolf, Schulz, Huber \& Plöckl, 1987). Its scattering geometry is shown in Fig. 2(b). For in-plane measurements ( $q_{\perp} \simeq 0$, corresponding to $\Phi_{0} \simeq 0$ ), the angle $\pi / 2-\Theta_{0}$ (the angle $\Theta_{0}$ is measured from the $z$ axis) corresponds to the Bragg angle $2 \theta_{B}$; out-of-plane measurements can be achieved by rotation of the detector around the $\Phi$ axis.

Although the diffractometers are similar in principle, there is one important difference between them.
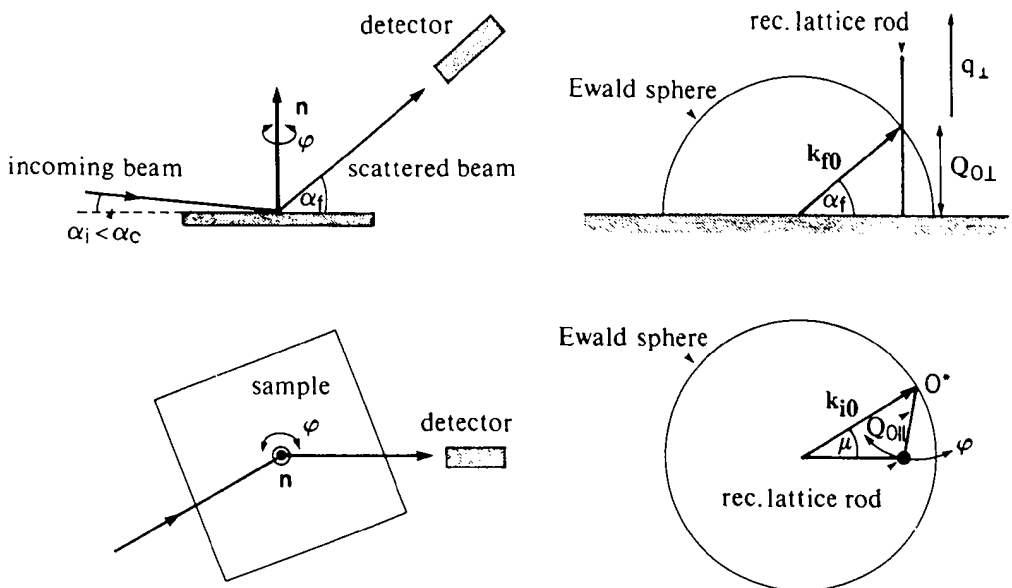

Fig. 1. Scattering geometry used for the measurement of superstructure intensities along $q_{\perp}$ shown in real (left) and reciprocal (right) space. The side view is shown in the upper part, the top view is shown in the lower part. The angles $\alpha_{i}$ and $\alpha_{f}$ denote the $X$-ray incidence angle and exit angle, respectively, $\mu$ is the in-plane scattering angle. The vector $\mathbf{n}$ is the surface normal. $Q_{0 \|}$ and $Q_{0 \perp}$ are the in-plane and out-of-plane components of the scattering vector $\mathbf{Q}_{0}$. The integrated intensity is measured by rotating the sample around its normal (i.e. the lattice rod around $0^{*}$, the origin of the reciprocal lattice) whilst keeping the detector fixed in the direction $\mathbf{k}_{f 0}$.

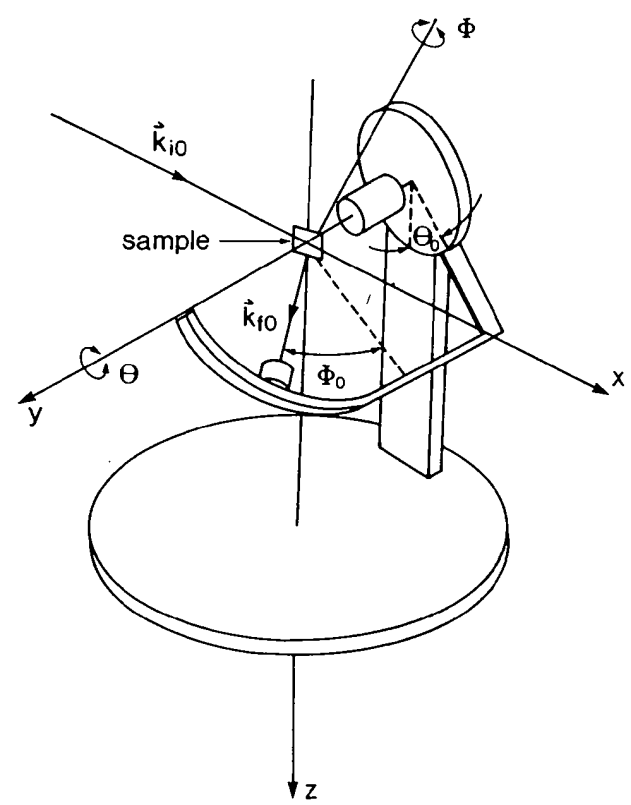

(a)

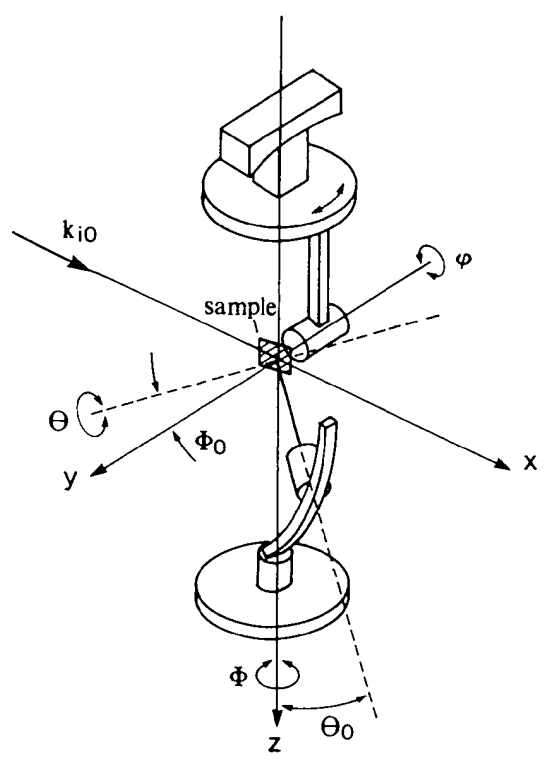

(b)

Fig. 2. $Z$-axis diffractometers used for the measurement of superlattice reflections. The primary beam is directed along the $x$ axis, the sample normal along the $y$ axis. (a) Type- $A$ diffractometer, $(b)$ type- $B$ diffractometer. In both cases, there are two degrees of freedom for the detector adjustment, an in-plane rotation axis, $\Theta$, and an axis rotating the detector out of plane, $\Phi$. 
Since, for the type- $A$ diffractometer, the in-plane rotation axis of the detector $(\Theta)$ is independent of the axis tilting the detector out of plane, the detector is rotated in a circle of constant $\alpha_{f}=\Phi_{0}$ around the surface normal. In contrast, for the type- $B$ diffractometer, the $\Theta$ axis is rotated by the $\Phi$ axis. The difference can be seen schematically in Fig. 3. This shows, for both diffractometer systems, the scattering geometry for an out-of-plane measurement in a stereographic projection to the sample surface $\left(q_{\perp}=0\right)$. The incoming beam, $\mathbf{k}_{i 0}$, is directed along the $x$ axis. The detector is rotated out of plane by the $\Phi$ axis, which is parallel to the $z$ axis of the coordinate frame corresponding to the standard settings of spherical coordinates. Since, for the type- $A$ diffractometer, the $\Theta$ axis normal to the plane of the paper (the $y$ axis) is independent of the $\Phi$ axis, the detector moves in a circle of constant $\alpha_{f}$ around the $\Theta$ axis (dotted circle). The finite resolution of the detector system is represented by an ellipse, whose principal axes indicate the width where the detector transmission has reduced to $1 / e$ of its maximum value (Yessik, Werner \& Sato, 1973; Boysen \& Adlhart,

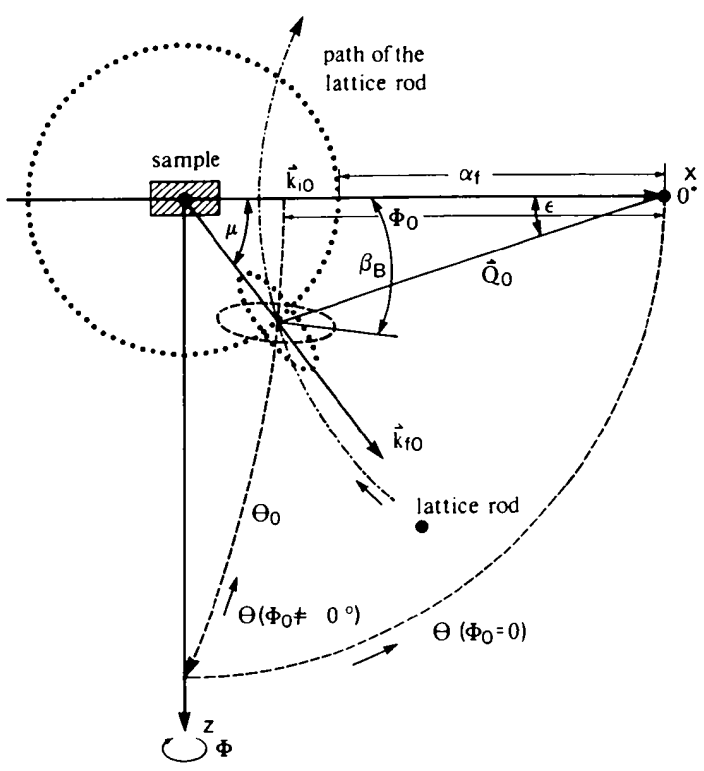

Fig. 3. Schematic drawing of the scattering geometry in reciprocal space showing the different detector settings of the $Z$-axis diffractometers in a stereographic projection onto the sample surface $\left(q_{\perp}=0\right)$. The detector systems whose resolutions are represented by ellipses (not on scale) are set to the out-of-plane reflection $\mathbf{Q}_{0}=\mathbf{k}_{i 0}-\mathbf{k}_{f 0}$. For the type- $A$ diffractometer, the detector rotates on a circle of $\alpha_{f}=$ constant (dotted line), whereas, for the type- $B$ diffractometer, the detector rotates on a great circle (dashed line) of the Ewald sphere. $\varepsilon$ is the in-plane projected angle between the vectors $Q_{0}$ and $-\mathbf{k}_{i 0}, \beta$ is the acute angle between the large axis of the ellipse and the primary beam (here shown for the type- $B$ diffractometer). For the type- $A$ diffractometer, $\beta=\mu$.
1987). In Fig. 3, both resolution functions are positioned at the reflection $\mathbf{Q}_{0}$ but not drawn to scale.

The situation is more complicated for the type- $B$ diffractometer, where the $\Theta$ axis is rotated by the $\Phi$ axis. Since the $\Phi$ axis is perpendicular to the $\Theta$ axis, the detector moves on a great circle of the Ewald sphere shown by the dashed lines in the projection to the $q_{1}=0$ plane. The beam exit angle $\alpha_{f}$ is related to $\Phi_{0}$ by $\sin \left(\alpha_{f}\right)=\sin \left(\Phi_{0}\right) \cos \left(\pi / 2-\Theta_{0}\right)$. (In contrast, for the type- $A$ diffractometer, we have $\Phi_{0}=\alpha_{f}$.)

The measured intensity data have to be corrected by the polarization factor $P\left(\mathbf{Q}_{0}\right)$ and the Lorentz factor $L\left(\mathbf{Q}_{0}\right)$. The latter describes the different velocities at which the reciprocal-lattice points are swept through the Ewald sphere depending on $\mathbf{Q}_{0}$. In the three-dimensional case, the size of the reciprocallattice points is so small that the total diffracted beam passes the slits in front of the detector. Lorentz correction factors depend on the scattering geometry and are discussed in Vol. II of International Tables for $X$-ray Crystallography (1959).

In surface X-ray diffraction, one important complication arises from the continuous distribution of the scattering intensity along $q_{\perp}$. The collected intensity additionally depends on $\Delta q_{\perp}$, the length of the rod accepted by the detector. For in-plane measurements, this is taken into account by multiplication of the intensity by the factor $\Delta q_{\perp}$.

The situation becomes complicated for out-ofplane measurements (rod scans) when the scattering plane is no longer parallel to the sample surface as shown in Fig. 1. Upon rotation of the lattice rod, the intersection of the rod with the Ewald sphere moves in a complicated way on the surface of the sphere and the measured intensity sensitively depends on the detector setting and the resolution. The differences between the detector arrangements of the two $Z$-axis diffractometers can also have consequences for the measured intensities, as is evident from Fig. 3.

For the type- $A$ diffractometer, the projection of the resolution ellipse onto the $q_{\perp}=0$ plane (dotted) is always aligned with one of its principal axes parallel to the direction of the (projected) exit beam. This does not apply for the type- $B$ diffractometer (dashed). Consequently, the path of the lattice rod through the detector resolution will be different.

Until now, only approximate solutions for the resolution correction have been used, which are only valid for small beam exit angles and large in-plane detector acceptances.

In this paper, we develop a general analytical expression for the resolution correction, $L\left(\mathbf{Q}_{0}\right)$, for rod scans measured in the $Z$-axis geometry considering both diffractometer systems. Additionally, we consider the effects on the measured intensity of: (i) the finite width of the superstructure reflections; (ii) the divergence of the primary beam; and (iii) an 
anisotropic transmission function, $T(\Delta \Theta, \Delta \Phi)$. The calculation is based on the assumption of (i) a grazing-incidence diffraction geometry, which is often used; (ii) a monochromatic primary beam of wavelength $\lambda_{0}$; and (iii) a negligible divergence of the primary beam along $q_{\perp}$. We derive the resolution correction for the more complicated case of the type- $B$ diffractometer. The simplifications leading to the resolution correction valid for the type- $A$ diffractometer are included in Appendix $A$.

\section{Calculation}

The primary-beam divergence is described by the distribution function $i\left(\mathbf{k}_{i}-\mathbf{k}_{i 0}\right)$, where $\mathbf{k}_{f}$ indicates a wave vector of the primary beam and $\mathbf{k}_{i 0}$ is its mean value. The distribution $i\left(\mathbf{k}_{i}-\mathbf{k}_{i 0}\right)$ is normalized:

$$
\int i\left(\mathbf{k}_{i}-\mathbf{k}_{i 0}\right) \mathrm{d} k_{i}^{3}=1 .
$$

The in-plane and out-of-plane divergences of the primary beam are given by the variables $\tau$ and $\sigma$, as shown in Fig. 4. With synchrotron radiation, where the sample is mounted vertically relative to the electron orbit, the parameter $\Delta \tau$ describes the $1 / e$ width of the intensity distribution with respect to $\tau$. Since $\Delta \tau$ is small, the volume element in $k$ space is, approximately,

$$
\mathrm{d} k_{i}^{3}=k_{i}^{2} \mathrm{~d} k_{i} \cos (\tau) \mathrm{d} \sigma \mathrm{d} \tau \simeq k_{i}^{2} \mathrm{~d} k_{i} \mathrm{~d} \sigma \mathrm{d} \tau .
$$

We assume a strictly monochromatic X-ray beam and a negligible out-of-plane divergence $(\Delta \sigma=0)$, since in grazing-incidence $\mathrm{X}$-ray diffraction experiments the (small) angle $\alpha_{i}$ must be well defined (i.e. $\Delta \sigma \ll \alpha_{i} \simeq$ $\alpha_{c} \simeq 0.3^{\circ}$.

We approximate the intensity distribution of the primary beam by a Gaussian function:

$$
i(\sigma, \tau, \lambda)=\delta(\sigma) \delta\left(\lambda-\lambda_{0}\right)\left(2 / \pi^{1 / 2} \Delta \tau\right) \exp (-2 \tau / \Delta \tau)^{2},
$$

where $\delta(\sigma)$ and $\delta\left(\lambda-\lambda_{0}\right)$ represent delta functions.

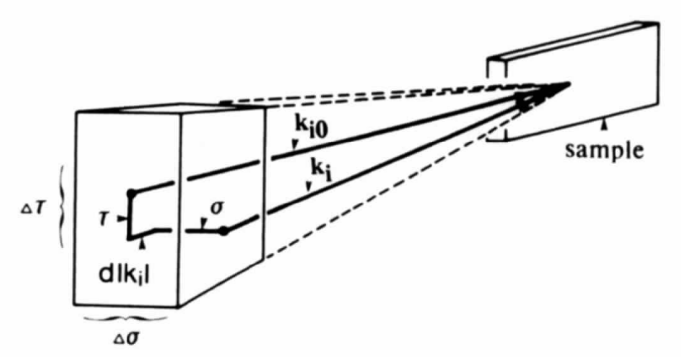

Fig. 4. Divergence of the primary beam parallel $(\Delta \tau)$ and perpendicular $(\Delta \sigma)$ to the sample surface. The centre of the primary beam is indicated by the wave vector $\mathbf{k}_{i 0}$. Deviations from $\mathbf{k}_{i 0}$ characterized by the wave vector $\mathbf{k}_{i}$ are due to $\Delta \tau$ and $\Delta \sigma$ as well as a wavelength shift $\mathrm{d} k_{i}$.
We consider in the following the scattering geometry of the type- $B$ diffractometer system shown in Fig. 5. The sample in the center of the Ewald sphere is illuminated by the primary beam, $\mathbf{k}_{i 0}$, at grazing incidence. It is directed along the $x$ axis. The sample normal $\mathbf{n}$ is parallel to the $y$ axis and the $z$ axis is perpendicular to both the $x$ axis and the $z$ axis (see Fig. 2). For a given reflection related to the momentum transfer $\mathbf{Q}_{0}=\left(\mathbf{k}_{f 0}-\mathbf{k}_{i 0}\right)$, the detector setting is given by the angles $\Theta_{0}$ and $\Phi_{0}$, where $\mathbf{k}_{f 0}$ is the wave vector of the scattered beam. In Appendix $A$, the relationships between the wave vector $\mathbf{k}_{f}$ and the angles $\Phi_{0}$ and $\Theta_{0}$ are derived. The anisotropic aperture of the detector system is represented by a resolution ellipse of angular acceptances $\Delta \Theta$ and $\Delta \Phi$, represented by the principal axes. With the assumption of a maximum angular acceptance of $2^{\circ}$, as realized in our detector system, the curvature along $\Delta \Theta$ and $\Delta \Phi$ on the Ewald sphere is still negligible. Because we use Soller slits in front of the detector (Soller, 1924; Stoecker \& Starbuck, 1965), to first order the transmission function of the detector is Gaussian:

$$
\begin{aligned}
T\left(\Theta-\Theta_{0}, \Phi-\Phi_{0}\right) \propto & \exp -\left[\left(\Theta-\Theta_{0}\right) / \Delta \Theta\right]^{2} \\
& \times \exp -\left[\left(\Phi-\Phi_{0}\right) / \Delta \Phi\right]^{2},
\end{aligned}
$$

which is normalized with respect to the solid angle:

$$
\Delta \Theta \Delta \Phi=\int T\left(\mathbf{k}_{f}-\mathbf{k}_{f 0}\right) \mathrm{d} k_{f}^{2} .
$$

For the measurement of the intensity related to $\mathbf{Q}_{0}$, the lattice rod is rotated around the origin of the reciprocal lattice, $0^{*}$, by the angular velocity $\partial \varphi / \partial t$.

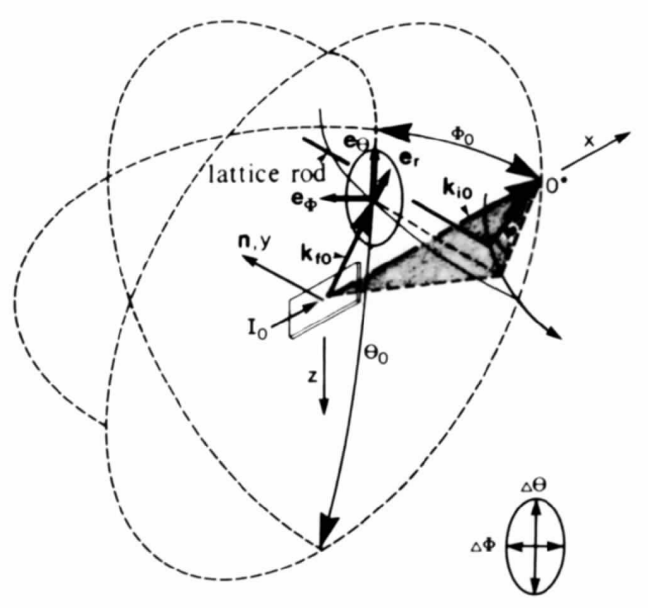

Fig. 5. Schematic view of the scattering geometry for the type- $B$ $Z$-axis diffractometer. During the $\varphi$ scan, the anisotropic resolution function of the detector (resolution $\Delta \Theta, \Delta \Phi$ ) is kept fixed at the angular positions $\Theta_{0}$ and $\Phi_{0}$ related to the scattered wave vector $\mathbf{k}_{f 0}$. The angle $\varphi$ indicates the in-plane angular deviation of the lattice rod from the scattering position, $\Theta_{0}, \Phi_{0}$. The path of the lattice rod is indicated by the arrow. 
This corresponds to the rotation of the sample by the in-plane angle, $\varphi$. The condition $\varphi=0$ corresponds to the point where the lattice rod intersects the Ewald sphere at $\mathbf{k}_{f 0}$, as indicated by the dashed lines.

The beam divergence and the instantaneous sample position $\varphi \neq 0$ during the scan lead to an actual scattering vector $\mathbf{Q}\left(\mathbf{k}_{i}, \varphi\right)$, which is different from $\mathbf{Q}_{0}$ :

$$
\mathbf{Q}\left(\mathbf{k}_{i}, \varphi\right)=\mathbf{Q}_{0}+\Delta \mathbf{Q}_{1}\left(\mathbf{k}_{i}-\mathbf{k}_{i 0}\right)+\Delta \mathbf{Q}_{2}(\varphi) .
$$

To calculate the integrated intensity at a given point $\varphi$ of the scan and for a given incidence vector $\mathbf{k}_{i}$, we have to integrate over the area of the detector by a variable $\mathbf{q}$ in reciprocal space:

$$
\begin{gathered}
I\left(\mathbf{k}_{i}, \varphi\right) \propto \int T\left[\mathbf{q}-\mathbf{Q}_{0}-\Delta \mathbf{Q}_{1}\left(\mathbf{k}_{i}-\mathbf{k}_{i 0}\right)\right. \\
\left.-\Delta \mathbf{Q}_{2}(\varphi)\right] \mathrm{d}^{3} \mathbf{q} .
\end{gathered}
$$

The reciprocal-lattice rods are broadened owing to the sample mosaic, the finite coherence length of the $X$-ray beam, the finite domain size and steps on the sample surface. However, in all practical cases, the domain size on the crystal surface, $\xi$, is much lower than the coherence length of the X-ray beam; therefore, we account for the lattice-rod broadening by considering the finite domain size.

Vlieg, van der Veen, Gurman, Norris \& MacDonald (1989), as well as Andrews \& Cowley (1985), have discussed in detail the effects of a finite correlation on the crystal surface on the reflection profiles. In many cases, a Gaussian correlation function is assumed, leading to a Gaussian broadening of the profiles. In the following, this is expressed by the function $D\left(\left|\mathbf{q}-\mathbf{Q}_{0}\right|\right)$.

For a given primary wave vector $\mathbf{k}_{i}$, a sample orientation $\varphi$ and a scattering vector $\mathbf{q}$, the measured intensity is proportional to:

$$
\begin{aligned}
& I\left(\mathbf{q}, \mathbf{k}_{i}, \varphi\right) \propto i\left(\mathbf{k}_{i}-\mathbf{k}_{i 0}\right)\left|F\left(\mathbf{Q}_{0}\right)\right|^{2} D\left(\left|\mathbf{q}-\mathbf{Q}_{0}\right|\right) \\
& \times T\left[\mathbf{q}-\mathbf{Q}_{0}-\Delta \mathbf{Q}_{1}\left(\mathbf{k}_{i}-\mathbf{k}_{i 0}\right)-\Delta \mathbf{Q}_{2}(\varphi)\right],
\end{aligned}
$$

where we have assumed that $|F(\mathbf{Q})|^{2}$ does not vary within $\Delta q_{\perp}$, the length of the rod that is accepted by the detector during the $\varphi$ scan.

The integrated intensity, $E\left(\mathbf{Q}_{0}\right)$, is obtained by integration over all wave vectors $\mathbf{k}_{i}$, all scattering vectors $\mathbf{q}$ and the crystal rotation angle $\varphi$ :

$$
\begin{aligned}
E\left(\mathbf{Q}_{0}\right)= & \iiint I\left(\mathbf{q}, \mathbf{k}_{i}, \varphi\right) \mathrm{d}^{3} \mathbf{q} \mathrm{d}^{3} \mathbf{k}_{i} \mathrm{~d} \varphi \\
= & {\left[n C\left|F\left(\mathbf{Q}_{0}\right)\right|^{2} / A_{E Z}^{2}\right] \iiint i\left(\mathbf{k}_{i}-\mathbf{k}_{i 0}\right) } \\
& \times D\left(\left|\mathbf{q}-\mathbf{Q}_{0}\right|\right) T\left[\mathbf{q}-\mathbf{Q}_{0}-\Delta \mathbf{Q}_{1}\left(\mathbf{k}_{i}-\mathbf{k}_{i 0}\right)\right. \\
& \left.-\Delta \mathbf{Q}_{2}(\varphi)\right] \mathrm{d}^{3} \mathbf{q} \mathrm{d}^{3} \mathbf{k}_{i} \mathrm{~d} \varphi .
\end{aligned}
$$

We have introduced $n=A / \xi^{2}$, the number of domains, because the profile function $D\left(\left|\mathbf{q}-\mathbf{Q}_{0}\right|\right)$ is normalized to the average area of the domains, $\xi^{2}$ : $\int D\left(\left|\mathbf{q}-\mathbf{Q}_{0}\right|\right) \mathrm{d} \mathbf{q} \propto \xi^{2}=A / n$, where $A$ is the area of the crystal surface illuminated by the $\mathrm{X}$-ray beam and $A_{E Z}$ corresponds to the area of the surface unit cell. The factor $C$ is defined by

$$
C=I_{0} P e^{4} /|\partial \varphi / \partial t| m^{2} c^{4}
$$

It takes into account $I_{0}$, the primary-beam intensity, the polarization factor $P$, the square of the electron radius and the angular velocity of the scan.

The integration in (9) can be performed by transformation into cylindrical coordinates, which leads to

$$
\begin{aligned}
E\left(\mathbf{Q}_{0}\right)= & {\left[4 C A\left|F\left(\mathbf{Q}_{0}\right)\right|^{2} \xi^{2} / \pi A_{E Z}^{2} \Delta \tau\right] } \\
& \times \int_{0}^{\infty} q_{\|} \exp -\left[\left(q_{\|}-Q_{0 \|}\right) \xi\right]^{2} \\
& \times \int_{-\infty}^{\infty} \exp -\left[(2 \tau / \Delta \tau)^{2}\right] \int_{-\infty}^{\infty} \exp -\left[\left(Q_{0 \|} \alpha \xi\right)^{2}\right] \\
& \times \int_{-\infty}^{\infty} \int_{0}^{\infty} T\left[q_{\|}-Q_{0 \|}+\Delta Q_{1 \|}(\tau),\right. \\
& \left.\alpha-\Delta \varphi_{1}(\tau)-\varphi, q_{\perp}-Q_{0 \perp}-\Delta Q_{1 \perp}(\tau)\right] \\
& \times \mathrm{d} q_{\perp} \mathrm{d} \varphi \mathrm{d} \alpha \mathrm{d} \tau q_{\|} \mathrm{d} q_{\|},
\end{aligned}
$$

where $\mathbf{q}$ is the integration variable in reciprocal space, $\mathbf{q}=\left(q_{\|}, \alpha, q_{\perp}\right)$, and $\mathbf{Q}_{0}$ is given by $\mathbf{Q}_{0}=\left(Q_{0 \|}\right.$, $\left.\alpha=0, Q_{0 \perp}\right)$.

The variables $\Delta Q_{1 \|}(\tau), \Delta \varphi_{1}(\tau)$ and $\Delta Q_{1 \perp}(\tau)$ are the corresponding correction terms resulting from beam divergence and $\Delta Q_{2}(\varphi)=(0, \varphi, 0)$ [see (6)]. Since we assume a monochromatic X-ray beam, the transmission is nonzero only on the Ewald sphere. Therefore, the integration over $q_{\perp}$ can be replaced by a projection onto the $q_{\perp}=0$ plane. The normalization of (5) remains valid for the projected transmission function and the parameters of the projected resolution ellipse can be calculated (see Appendix $A$ ).

After integration of (11), we finally obtain for the integrated intensity:

$$
E\left(\mathbf{Q}_{0}\right)=\left[2 \pi C\left|F\left(\mathbf{Q}_{0}\right)\right|^{2} \lambda_{0} A / A_{E Z}^{2}\right] L\left(\mathbf{Q}_{0}\right),
$$

where:

$$
\begin{gathered}
L\left(\mathbf{Q}_{0}\right)=L_{1}\left(\mathbf{Q}_{0}\right) L_{2}\left(\mathbf{Q}_{0}\right) \\
L_{1}\left(\mathbf{Q}_{0}\right)=\Delta \Theta \Delta \Phi / d^{1 / 2} a b Q_{0 \|} \\
L_{2}\left(\mathbf{Q}_{0}\right)=\left\{1+\lambda_{0}^{2}\left[e-\left(c^{2} / d\right)\right]\left[\Delta \tau^{2} \Gamma^{2}+\left(1 / \xi^{2}\right)\right]\right\}^{-1 / 2}
\end{gathered}
$$

The parameters $a, b, c, d, e$ and $\Gamma$ are geometrical parameters that also contain the differences between the resolution corrections for the type- $A$ and type- $B$ diffractometers. These are listed in Appendix $A$.

Equations (12) to (15) represent the result of the calculation, which is valid for both detector systems 
(except for the different meanings of the geometrical parameters).

The parameter $L\left(\mathbf{Q}_{0}\right)$ is the correction to the measured integrated intensity, $E\left(\mathbf{Q}_{0}\right)$, owing to the resolution of the detector system. It can be factorized into two components, $L_{1}\left(\mathbf{Q}_{0}\right)$ and $L_{2}\left(\mathbf{Q}_{0}\right)$, the first describing the resolution correction assuming a strictly parallel primary beam, $\Delta \tau=0$, and a delta-function-like lattice rod. This gives $L_{2}\left(\mathbf{Q}_{0}\right)=1$. The second term takes into account the primary-beam divergence $\Delta \tau$ and the lattice-rod broadening due to the finite domain size, $\xi$.

In Appendix $B$, we show that the general expression for the integrated intensity for $q_{\perp} \rightarrow 0$ is equivalent to the small $\alpha_{f}$ approximation (Feidenhans'l, 1989).

\section{Discussion}

\section{A. Detector setting}

In order to elucidate the different effects contributing to the total intensity correction factor, we have calculated $L\left(\mathbf{Q}_{0}\right)$ for the $(1 / 20)$ and the $(1 / 22)$ superlattice reflection rod of the $\operatorname{Ge}(001)(2 \times 1)$ structure that has been investigated recently (Rossmann, Meyerheim, Jahns, Wever, Moritz, Wolf, Dornisch \& Schulz, 1992). The indices are related to the tetragonal setting of the unit cell $\left(a_{0}=b_{0}=4.000\right.$, $c_{0}=5.658 \AA$ ) and the wavelength is $\lambda_{0}=1.54 \AA$. First, we assume ideal experimental conditions corresponding to $L_{2}\left(\mathbf{Q}_{0}\right)=1$. This means that the primary beam is strictly parallel and the superlattice rods are represented laterally by a two-dimensional $\delta$ function.

In Fig. 6, the resolution correction term $L_{1}\left(\mathbf{Q}_{0}\right)$ is shown for both the type- $A$ diffractometer (dotted lines) and the type- $B$ diffractometer (dashed lines). Two detector settings can be considered. The first corresponds to high resolution in plane $\left(\Delta q_{\|}\right)$and low resolution out of plane $\left(\Delta q_{\perp}\right)$. This case is schematically shown by the ellipse. In the following, we use $\Delta \Theta=0.4^{\circ}=\Delta q_{\|}=4.53 \times 10^{-3} \AA^{-1}$ and $\Delta \Phi=$ $2.0^{\circ}=\Delta q_{\perp}=2.26 \times 10^{-2} \AA^{-1}$, which approximately corresponds to the experimental conditions of our diffractometer setup, where Soller slits are used in front of the detector. In the second setting, the resolution ellipse is rotated by $90^{\circ}$ relative to the first, leading to low resolution in plane and high resolution out of plane (not shown).

The detector setting shown is chosen mostly for the measurement of in-plane reflections $\left(q_{\perp} \simeq 0\right)$, in order to maximize the measured intensity, since in this case $E\left(\mathbf{Q}_{0}\right) \propto \Delta q_{\perp}$. The second detector setting has the advantage that broad reflections are completely accepted by the detector that would otherwise be cut off when using a high in-plane resolution. Further, $\Delta q_{\perp}$ has to be kept small enough for an accurate intensity measurement, since the intensity may vary rapidly along $q_{\perp}$.

It should be noted that the resolutions specified above are valid for in-plane measurements only. For out-of-plane data collection, the resolutions change considerably as functions of $q_{\perp}$. This is expressed by the variation of $L_{1}\left(\mathbf{Q}_{0}\right)$.

\section{B. Effect of the detector geometry}

As shown by the calculation in Fig. 6, in most cases there are only minor differences between the intensity corrections corresponding to the type- $A$ (dotted lines) and type- $B$ (dashed lines) $Z$-axis diffractometers. However, at very large $q_{\perp}$, there may be differences as large as a factor of two. For example, consider the $(1 / 22)$ rod for $q_{\perp}>3.2$ reciprocal-lattice units. This is because the path of the lattice rod through the resolution function is correspondingly larger for the type- $A$ setup, as can also be seen in Fig. 3.

We have additionally plotted, as a solid line, the resolution correction for the $(1 / 20)$ rod using the small $\alpha_{f}$ approximation discussed in Appendix B. Although it is strictly valid for $q_{\perp}=0$ only, there is good agreement with our solution up to about $q_{\perp} \simeq$ $1.5 c^{*}$, where $c^{*}=1 / c_{0}=0.177 \AA^{-1}$ is the reciprocallattice unit normal to the sample surface. However, at larger $q_{\perp}$, the approximation breaks down.

Since, in general, the detector resolution should be aligned such as to maximize the measured intensity, the (anisotropic) detector function should be aligned to maximize the path of the rod through it. Increasing

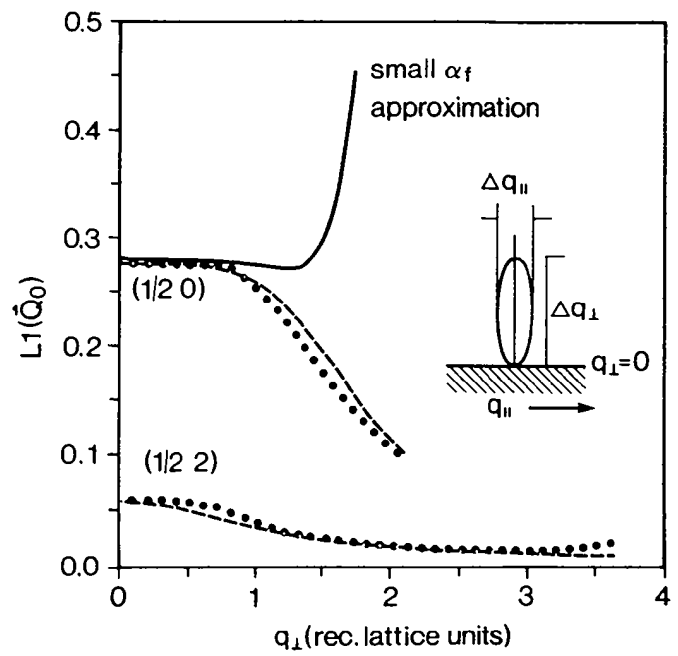

Fig. 6. Resolution correction factor $L_{1}\left(Q_{0}\right)$ calculated for the $(1 / 20)$ and $(1 / 22)$ rods of the $\mathrm{Ge}(001)(2 \times 1)$ reconstruction $\left(\lambda_{0}=1.54 \AA\right)$. The dotted line corresponds to the type- $A$ diffractometer, the dashed line to the type- $B$ diffractometer. The resolution of the detector system is $\Delta q_{\|}=4.53 \times 10^{-3} \AA^{-1}$ and $\Delta q_{\perp}=2.26 \times 10^{-2} \AA^{-1}$ (at $\left.q_{\perp}=0\right)$, as is schematically represented by the ellipse. The small $\alpha_{f}$ approximation is represented by the solid line. 
$\Delta q_{\perp}$ and $\Delta q_{\|}$is not always possible owing to background and resolution requirements. Therefore, for both diffractometers, it would be advantageous to rotate the detector system as a function of $q_{\perp}$. However, this is, practically, not always feasible (note that, using a diffractometer setup where $\alpha_{f}$ is constant but $\alpha_{i}$ is varied in order to vary $q_{\perp}$, the detector resolution is constant).

For the optimum choice of detector setting for out-of-plane measurements, it is important to not only consider how to maximize the measured intensity but also to take into account sources of systematic errors as well. For example, a small misalignment of the detector along $q_{\perp}$ is less important if the correction function is only slowly varying with $q_{\perp}$. Besides the detector setting relative to the path of the lattice rod, the $1 / Q_{0 \|}$ dependency of $L_{1}\left(Q_{0}\right)$ is the most important factor contributing to the measured intensity. Usually, the $1 / \sin 2 \theta_{B}$ (see Fig. 6) dependency is referred to as the Lorentz factor, where $\sin \theta_{B}=0.5 \lambda_{0}\left|\mathbf{Q}_{0}\right|$ and $\theta_{B}$ is the Bragg angle of the reflection.

\section{Finite-size effects}

So far, we have confined the discussion to ideal experimental conditions. Superstructure reflections have a finite width, owing to the domain size, $\xi$, mosaic spread and steps on the surface. This leads to the broadening of the lattice rod as discussed by Vlieg et al. (1989). This is accounted for by the factor $L_{2}\left(\mathbf{Q}_{0}\right)<1$.

Besides the high primary-beam intensity, synchrotron radiation is advantageous owing to its inherent parallelity in the plane of the electron orbit [see, for example, Koch, Eastman \& Farge (1983)]. This corresponds to a small in-plane divergence $(\Delta \tau<$ $0.01^{\circ}$ ), since the sample is mounted vertically. The out-of-plane collimation is achieved by slits in front of the sample. It is kept very small $\left(\Delta \sigma<0.05^{\circ}\right)$. Therefore, we only consider the lattice-rod broadening caused by the domain size, mosaic spread and steps. For simplicity, we chose the domain size $\xi$ as the representative parameter. Taking into account the in-plane divergence $\Delta \tau$ of the primary beam does not change the basic behaviour of $L_{2}\left(\mathbf{Q}_{0}\right)$, since it corresponds to an 'effective' broadening of the lattice rod.

We confine our discussion to the type- $B$ diffractometer.

In Fig. 7 , we show the function $L_{2}\left(\mathbf{Q}_{0}\right)$ of the $(1 / 20)$ and the $(1 / 22)$ rods for both resolution settings. Two domain sizes are considered, $\xi=100 \AA$ (dashed lines) and $\xi=40$ ก $\AA$ (solid lines) corresponding to bad and reasonable quality surfaces, respectively [see, for example, Vlieg et al. (1989)].

The effect of the domain size on the resolution correction is dramatic. Considering the usual setting for the in-plane data collection (Fig. 7a), the integrated intensity $\left[E\left(\mathbf{Q}_{0}\right) \propto L_{1}\left(\mathbf{Q}_{0}\right) L_{2}\left(\mathbf{Q}_{0}\right)\right]$ at $q_{\perp}=0$ is reduced by more than $50 \%$ for $\xi=100 \AA$, since the lateral resolution of the detector setting is too high to collect the whole width of the rod. Even in the case of $\xi=400 \AA$, the effect is still about $15 \%$ at $q_{\perp}=0$. Relaxing the lateral resolution may solve this problem, provided the background remains low. This can be seen in Fig. $7(b)$, showing $L_{2}\left(\mathbf{Q}_{0}\right)$ for the opposite setting of the resolution function. At low $q_{\perp}$, there is at most a $10 \%$ intensity reduction even for the $\xi=100 \AA$ sample.

However, the more important complication arises from the variation of $L_{2}\left(\mathbf{Q}_{0}\right)$ as a function of $q_{1}$, which is due to the changing path of the reflection through the resolution ellipse. As can be seen, there may be a variation by up to $100 \%$ along the rod. In summary, consideration of the sample quality is a prerequisite for the proper resolution correction.
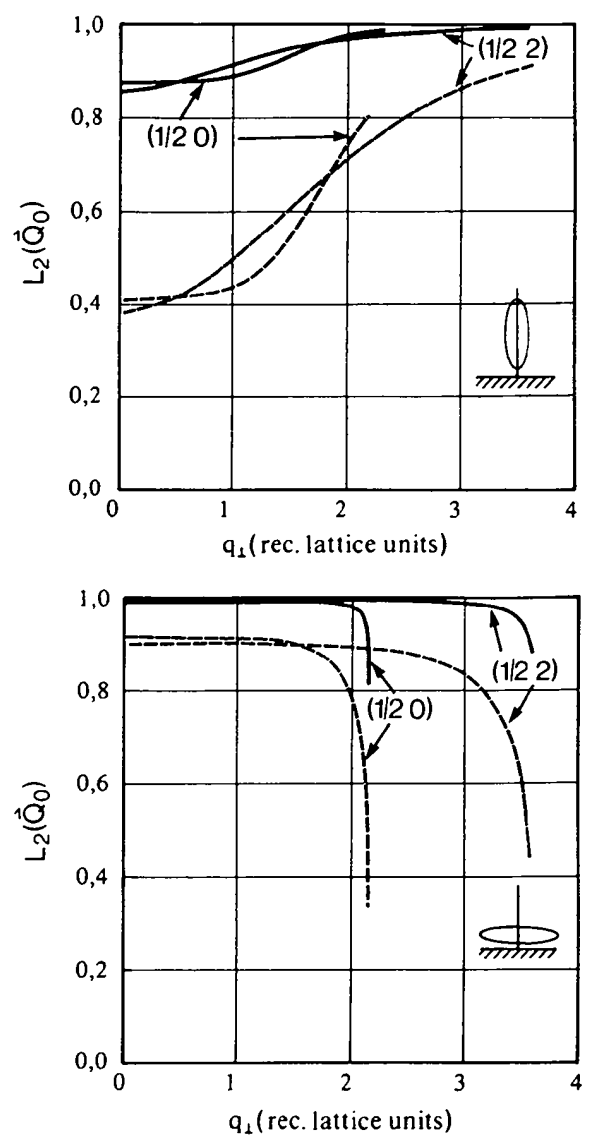

Fig. 7. $L_{2}\left(\mathbf{Q}_{0}\right)$ calculated for the type- $B$ diffractometer versus $q_{\perp}$ calculated for the $(1 / 20)$ and $(1 / 22)$ rods, assuming an average domain size of $\xi=400 \AA$ (solid lines) and $\xi=100 \AA$ (dashed lines). The beam divergence is neglected $(\Delta \tau=0)$. Both settings of the resolution function (at $q_{1}=0$ ) are considered as shown schematically by the ellipses. Depending on the detector setting and the domain size, there may be a reduction of the measured intensity of more than $50 \%$. 


\section{Summary}

For two types of $Z$-axis diffractometer, we have developed an analytical expression for the detector resolution correction, $L\left(\mathbf{Q}_{0}\right)$, which must be applied for the analysis of out-of-plane intensity data obtained from superstructures on crystal surfaces.

The calculation of $L\left(\mathbf{Q}_{0}\right)$, i.e. the convolution of the intrinsic reflection profile with the resolution function, is generally a three-dimensional problem. If a monochromatic X-ray beam is assumed, this can be reduced to two dimensions by projection onto the sample surface $\left(q_{\perp}=0\right)$. The correction factor could be factorized, $L\left(\mathbf{Q}_{0}\right)=L_{1}\left(\mathbf{Q}_{0}\right) L_{2}\left(\mathbf{Q}_{0}\right)$, the first term assuming a strictly parallel beam and a delta-functionlike lateral lattice-rod profile, the second term considering the lattice-rod broadening that is due to beam divergence and sample imperfections. Choosing the experimental parameters characteristic for surface $\mathrm{X}$-ray scattering experiments, we have calculated $L_{1}\left(\mathbf{Q}_{0}\right)$ and $L_{2}\left(\mathbf{Q}_{0}\right)$ for the $(1 / 20)$ and the $(1 / 22) \operatorname{rod}$ of the $\mathrm{Ge}(001)(2 \times 1)$ reconstruction.

In some special cases, the resolution correction $L_{1}\left(\mathbf{Q}_{0}\right)$ corresponding to the type- $A$ diffractometer may differ considerably from $L_{1}\left(\mathbf{Q}_{0}\right)$ of the type- $B$ diffractometer. This is due to the different detector orientations relative to the scattered beam.

It could be shown that the small $\alpha_{f}$ approximation $L_{1}\left(\mathbf{Q}_{0}\right) \propto \Delta q_{\perp}$ is valid up to about $q_{\perp}=1.5 c^{*}$ $\left(c^{*}=1 / c_{0}=0.177 \AA^{-1}\right.$, corresponding to the germanium lattice parameter). However, at larger $q_{\perp}$, it deviates considerably. This is because, during the $\varphi$ scan, the path of the reflection within the resolution ellipse varies as a function of $q_{\perp}$.

Depending on the setting of the detector system and $q_{\perp}$, the finite width of the lattice rod may significantly reduce the measured intensity, since it is no longer completely accepted by the aperture of the detector. Therefore, in particular, the consideration of the sample quality is an additional prerequisite for obtaining reliable intensity data in rod-scan experiments.

The authors thank R. Wunderlich for carefully preparing the figures. This work is supported by the Bundesministerium für Forschung und Technologie under project no. 05464 IAB 8.

\section{APPENDIX $A$ \\ Geometric parameters}

Fig. 8 shows, for the type- $B$ diffractometer, the experimental geometry used for out-of-plane measurements (see also Fig. 3). The scattering vector $\mathbf{Q}_{0}$ can be separated into $Q_{0 \|}$ and $Q_{0 \perp}$, corresponding to its in-plane and out-of-plane components, respectively. The wave vector of the scattered beam, $\mathbf{k}_{f 0}$, is related to the angles $\Theta_{0}$ and $\Phi_{0}$ where, for the type- $B$ diffractometer, the $\Theta$ axis is rotated by the $\Phi$ axis. Note that, for the measurement of in-plane reflections $(\Phi \simeq 0)$, the angle $\pi / 2-\Theta_{0}$ corresponds to $2 \theta_{B}$, where $\theta_{B}$ is the Bragg angle of the reflection.

From the cosine law, the in-plane scattering angle $\mu$ and the angle $\varepsilon$ can be expressed by

$$
\begin{gathered}
\varepsilon=\arccos \left(\lambda_{0}\left|\mathbf{Q}_{0}\right|^{2} / 2 Q_{0 \|}\right) \\
\mu=\arccos \lambda_{0}\left[\left(2 / \lambda_{0}^{2}\right)-\left|\mathbf{Q}_{0}\right|^{2}\right] / 2\left[\left(1 / \lambda_{0}^{2}\right)-Q_{0 \perp}^{2}\right]^{1 / 2} .
\end{gathered}
$$

Further, the angles $\Theta_{0}$ and $\Phi_{0}$ can be derived by the following trigonometric relations:

$$
\begin{aligned}
\sin \left(\Phi_{0}\right) & =\sin \left(\alpha_{f}\right) / \cos \left[(\pi / 2)-\Theta_{0}\right] \\
\sin \left(\alpha_{f}\right) & =Q_{0 \perp} \lambda_{0},
\end{aligned}
$$

where, for large beam exit angles and grazing incidence, we have the approximation $\sin \alpha_{i} \simeq 0$. Remember also that $Q_{0 \perp}=\left|\mathbf{k}_{f 0}\right| \sin \alpha_{f}$.

The angle $\Theta_{0}$ is given by

$$
\Theta_{0}=\arccos \left[1-\cos ^{2}\left(2 \theta_{B}\right)-\cos ^{2}\left[(\pi / 2)-\alpha_{f}\right]^{1 / 2}\right. \text {. }
$$

In the following, we quote the meanings of the geometric parameters that are needed to evaluate (12)-(15). First, we confine ourselves to the type- $B$ diffractometer.

$$
a=\left(\delta_{+}+\delta_{-}\right) / 2, \quad b=\left(\delta_{+}-\delta_{-}\right) / 2,
$$

where

$$
\delta_{ \pm}=\left(d_{\Theta}^{2}+d_{\Phi}^{2} \pm 2 d_{\Theta} d_{\Phi} \sin \gamma\right)^{1 / 2}
$$

and

$$
\begin{aligned}
& d_{\Theta}=\Delta \Theta\left(1-\cos ^{2} \Theta_{0} \sin ^{2} \Phi_{0}\right)^{1 / 2}, \\
& d_{\Phi}=\Delta \Phi\left|\sin \Phi_{0}\right| .
\end{aligned}
$$

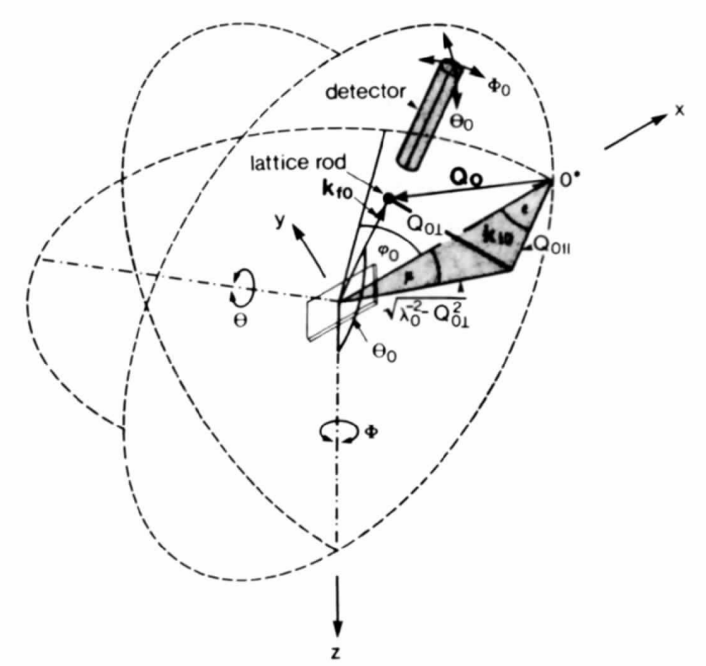

Fig. 8. Scattering geometry for out-of-plane measurements (type- $B$ diffractometer), indicating the experimental parameters $\Theta_{0}, \Phi_{0}, \varepsilon$ and $\mu$. The sample normal is parallel to the $y$ axis. 
$d_{\Theta} / \lambda_{0}$ and $d_{\Phi} / \lambda_{0}$ are the conjugated diameters of the projected resolution ellipse; $a / \lambda_{0}$ and $b / \lambda_{0}$ represent its principal axes related to the conjugated diameters by the Appolonius law of the ellipse geometry.

The (acute) angle $\gamma$ between the conjugated diameters can be calculated by

$\gamma=\arccos \left[\cos \Theta_{0} \cos \Phi_{0} /\left(1-\cos ^{2} \Theta_{0} \sin ^{2} \Phi_{0}\right)^{1 / 2}\right]$.

Further, we have, for $c, d$ and $e$,

$$
\begin{aligned}
& c=\cos (\beta-\varepsilon) \sin (\beta-\varepsilon)\left[\left(1 / a^{2}\right)-\left(1 / b^{2}\right)\right], \\
& d=\left[\sin ^{2}(\beta-\varepsilon) / a^{2}\right]+\left[\cos ^{2}(\beta-\varepsilon) / b^{2}\right], \\
& e=\left[\cos ^{2}(\beta-\varepsilon) / a^{2}\right]+\left[\sin ^{2}(\beta-\varepsilon) / b^{2}\right],
\end{aligned}
$$

where the angle $\varepsilon$ is given by $(A 1)$ and $\beta$ is the (always acute) angle between the long principal axis of the projected ellipse and the primary beam, $\mathbf{k}_{i 0}$ (see, for example, Fig. 3):

$$
|\beta|=\arcsin \left\{\left(b / d_{\Phi}\right)\left[\left(a^{2}-d_{\Phi}^{2}\right) /\left(a^{2}-b^{2}\right)\right]^{1 / 2}\right\} .
$$

For the type-A diffractometer, we derive, from Fig. 9 , the following (simpler) relations:

$$
\beta=\pi / 2-\mu, \quad a=\Delta \Theta, \quad b=\Delta \Phi \sin \alpha_{f}
$$

or

$$
\beta=\mu, \quad b=\Delta \Theta, \quad a=\Delta \Phi \sin \alpha_{f},
$$

depending on the ellipse orientation, since we have to satisfy the condition $a>b$. The other equations can then also be used.

Finally, we have, for $\Gamma$,

$$
\Gamma=\left(\cos \mu / \lambda_{0}\right)-Q_{0 \|} \cos (\mu+\varepsilon) .
$$

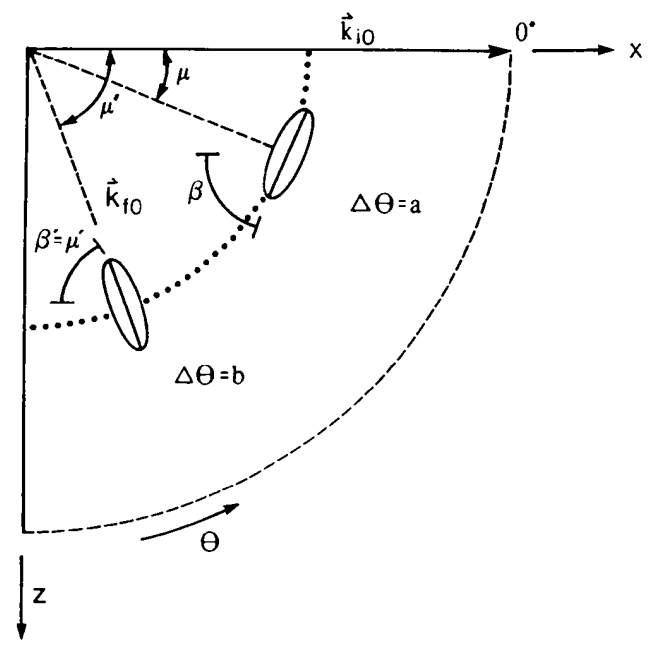

Fig. 9. Scattering geometry for the type- $A$ diffractometer shown for both possible orientations of the projected resolution ellipse. Note that, depending on the setting, we have $\beta=\pi / 2-\mu$ and $\Delta \Theta=a$ or $\beta=\mu$ and $\Delta \Theta=b$.

\section{APPENDIX $B$}

\section{Resolution-correction measurements $q_{\perp} \rightarrow 0$}

We derive the approximation for the resolution correction for the limiting case $q_{\perp} \rightarrow 0$. It is valid for both diffractometer geometries. In the $q_{\perp} \rightarrow 0$ limit corresponding to $\Phi_{0} \rightarrow 0$, the principal axis $\Delta \Phi / \lambda_{0}$ of the resolution ellipse is oriented normal to the $q_{\perp}=0$ plane (i.e. parallel to the lattice rods). Therefore, we have, for the length of the rod accepted by the detector, $\Delta q_{\perp}=\Delta \Phi / \lambda_{0}$. Further, the principal axes of the projected resolution ellipse are given by $b=0$ and $a=\Delta \Theta$, meaning that it degenerates to a line as shown in Fig. 10. For in-plane reflections, the Bragg condition is

$$
\left|Q_{0}\right|=\left|Q_{0 \|}\right|=\left(2 / \lambda_{0}\right) \sin \theta_{B},
$$

where $\theta_{B}=|\beta-\varepsilon|$, so that, using the definition of the parameter $d$ in $(A 10)$, we can write

$$
d^{1 / 2} b=\left[\left(b^{2} / a^{2}\right) \sin ^{2}\left(\theta_{B}\right)+\cos ^{2}\left(\theta_{B}\right)\right]^{1 / 2}=\cos \left(\theta_{B}\right),
$$

since $b \rightarrow 0$ for $q_{1} \rightarrow 0$.

Correspondingly, we can write for the integrated intensity, $E\left(\mathbf{Q}_{0}\right)[(11)]$, assuming $L_{2}\left(\mathbf{Q}_{0}\right)=1$ :

$$
\begin{aligned}
E\left(\mathbf{Q}_{0}\right)= & {\left[2 \pi C\left|F\left(\mathbf{Q}_{0}\right)\right|^{2} A / A_{E Z}^{2}\right] } \\
& \times\left[\lambda_{0}^{3} / 2 \sin \left(\theta_{B}\right) \cos \left(\theta_{B}\right)\right] \Delta q_{\perp},
\end{aligned}
$$

which is identical to the expression given by Feidenhans'l (1989). This applies for both type- $A$ and type- $B$ diffractometers.

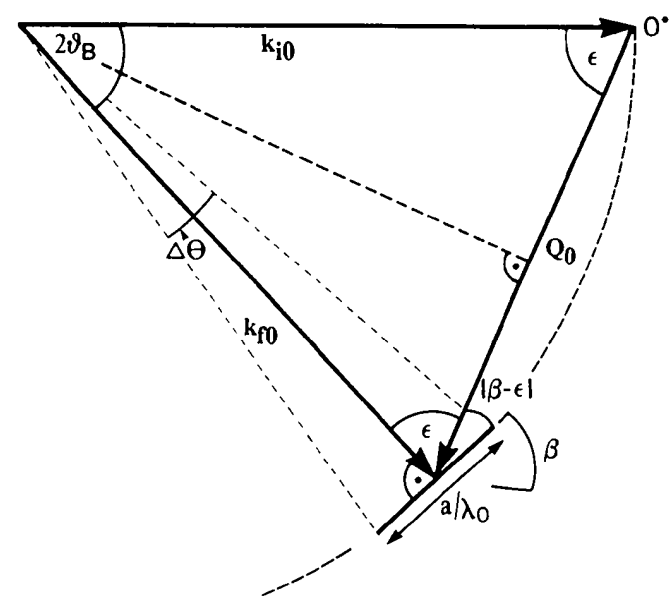

Fig. 10. Scattering geometry for in-plane reflections. The projected resolution ellipse has degenerated to a line corresponding to the large principal axis $a / \hat{\lambda}_{0}=\Delta \Theta / \hat{\lambda}_{0}$. The resolution corrections of the two diffractometers are identical in the $q_{\perp} \rightarrow 0$ limit. 


\begin{tabular}{|c|c|}
\hline \multicolumn{2}{|c|}{$\begin{array}{c}\text { APPENDIX } C \\
\text { List of some important parameters }\end{array}$} \\
\hline $\begin{array}{l}\lambda_{0} \\
A\end{array}$ & $\begin{array}{l}\text { Wavelength of the X-ray beam } \\
\text { Area of the crystal surface } \\
\text { illuminated by the X-ray beam }\end{array}$ \\
\hline$A_{E Z}$ & Area of the surface unit cell \\
\hline$n$ & $\begin{array}{l}\text { Number of coherently scattering } \\
\text { domains }\end{array}$ \\
\hline a & Surface normal \\
\hline$\xi^{2}$ & Average domain size $\left(\AA^{2}\right)$ \\
\hline$\Delta \tau$ & In-plane primary-beam divergence \\
\hline$\Delta \sigma$ & $\begin{array}{l}\text { Out-of-plane primary-beam } \\
\text { divergence }\end{array}$ \\
\hline $\begin{array}{l}\mathbf{k}_{i 0} \\
\mathbf{k}_{f 0}\end{array}$ & $\begin{array}{l}\text { (Central) primary-beam wave vector } \\
\text { (Central) scattered-beam wave } \\
\text { vector, }\left|\mathbf{k}_{f 0}\right|=\left|\mathbf{k}_{i 0}\right|=1 / \lambda_{0}\end{array}$ \\
\hline $\begin{array}{l}\mathbf{Q}_{0} \\
\Theta_{0}, \Phi_{0}\end{array}$ & $\begin{array}{l}\text { Scattering vector: } \mathbf{Q}_{0}=\mathbf{k}_{f 0}-\mathbf{k}_{i 0} \\
\text { Detector's position corresponding } \\
\text { to } \mathbf{k}_{f 0}\end{array}$ \\
\hline$\Delta \Theta, \Delta \Phi$ & Angular resolution of the detector \\
\hline$T(\Delta \Theta, \Delta \Phi)$ & Transmission function of the detector \\
\hline $\mathbf{k}_{i}, \mathbf{k}_{f}$ & $\begin{array}{l}\text { Wave vector within the primary and } \\
\text { scattered beam }\end{array}$ \\
\hline $\mathbf{q}=\left(q_{\|}, \alpha, q_{\perp}\right)$ & $\begin{array}{l}\text { Reciprocal-space variable in } \\
\text { cylindrical coordinates }\end{array}$ \\
\hline$\alpha$ & $\begin{array}{l}\text { Angular component of } \mathbf{q} \text { in } \\
\text { cylindrical coordinates }\end{array}$ \\
\hline$\varphi$ & $\begin{array}{l}\text { Angular sample position during scan: } \\
\varphi=0 \Leftrightarrow \mathbf{Q}_{0}\end{array}$ \\
\hline$\Delta \mathbf{Q}_{1}\left(\mathbf{k}_{i}-\mathbf{k}_{0}\right)$ & $\begin{array}{l}\text { Correction to } \mathbf{Q}_{0} \text { due to the primary- } \\
\text { beam divergence }\end{array}$ \\
\hline$\Delta \mathbf{Q}_{2}(\varphi)$ & $\begin{array}{l}\text { Correction to } \mathbf{Q}_{0} \text { due to actual } \\
\text { sample position }\end{array}$ \\
\hline $\mathbf{Q}\left(\mathbf{k}_{i}, \varphi\right)$ & $\begin{array}{l}\text { Resulting scattering vector, } \\
\qquad \begin{array}{l}\mathbf{Q}\left(\mathbf{k}_{i}, \varphi\right)=\mathbf{Q}_{0}+\Delta \mathbf{Q}_{1}\left(\mathbf{k}_{f}-\mathbf{k}_{0}\right) \\
+\Delta \mathbf{Q}_{2}(\varphi)\end{array}\end{array}$ \\
\hline
\end{tabular}

\begin{tabular}{|c|c|}
\hline$a / \lambda_{0}, b / \lambda_{0}$ & $\begin{array}{l}\text { Principal axes of the projected } \\
\text { resolution ellipse }\end{array}$ \\
\hline$\beta$ & $\begin{array}{l}\text { Acute angle between the large } \\
\text { principal axes of the projected } \\
\text { resolution ellipse and } \mathbf{k}_{i 0}\end{array}$ \\
\hline$\varepsilon$ & Angle between $Q_{0 \|}$ and $-\mathbf{k}_{i 0}$ \\
\hline & In-plane scattering angle \\
\hline$\theta_{B}$ & Bragg angle related to $\mathbf{Q}_{0}$ \\
\hline
\end{tabular}

\section{References}

Andrews, S. R. \& Cowley, R. A. (1985). J. Phys. C, 18, 6427-6439.

Bloch, J. M. (1985). J. Appl. Cryst. 18, 33-36.

Boysen, H. \& Adlhart, W. (1987). J. Appl. Cryst. 20, 200-209.

Brennan, S. \& Eisenberger, P. (1984). Nucl. Instrum. Methods, A222, 164-167.

Feidenhans'L (1989). Surf. Sci. Rep. 10, 105-188.

International Tables for X-ray Crystallography (1959), Vol. II. Birmingham: Kynoch Press. (Present distributor Kluwer Academic Publishers, Dordrecht.)

Koch, E. E., Eastman, D. E. \& Farge, Y. (1983). Handbook on Synchrotron Radiation, Vol. IA, edited by E. Косн, pp. 1-59. Amsterdam: North-Holland.

Kretschmar, F., Wolf, D., Schulz, H., Huber, H. \& PLOCCKL, H. (1987). Z. Kristallogr. 178, 130-132.

RoBinson, I. K. (1991). Handbook on Synchrotron Radiation, Vol. III, edited by G. S. Brown \& D. E. Moncton, pp. 221-266. Amsterdam: North-Holland.

Rossmann, R., Meyerheim, H. L., Jahns, V., Wever, J., Moritz, W., Wolf, D., Dornisch, D. \& Schulz, H. (1992). Surf. Sci. 279, 199-209.

SOlLER, W. (1924). Phys. Rev. 24, 158-167.

Stoecker, W. C. \& Starbuck, J. W. (1965). Rev. Sci. Instrum. 36, 1953-1958.

Vlieg, J. F., van der Veen, J. F., Gurman, S. J., Norris, C. \& MacDonald, J. E. (1989). Surf. Sci. 210, 301-321.

Yessik, M., Werner, S. A. \& Sato, H. (1973). Acta Cryst. A29, 372-382. 